\title{
Impact of baseline patient characteristics on dupilumab efficacy in type 2 asthma
}

\begin{abstract}
To the Editor:
Copyright (CThe authors 2021.

This version is distributed under the terms of the Creative Commons Attribution NonCommercial Licence 4.0. For commercial reproduction rights and permissions contact permissions@ersnet.org

Received: 22 Dec 2020 Accepted: 23 May 2021

\section{○@®@}

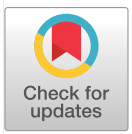

Severe asthma affects an estimated $5-10 \%$ of the total asthma patient population [1]. Various demographic factors, such as sex, age, obesity and age of onset, have been associated with asthma disease severity $[2,3]$, and the efficacy of asthma treatments has previously been found to vary depending on patient demographics $[4,5]$.

Approximately $50 \%$ of asthma patients are affected by type 2 inflammatory asthma, characterised by increased production of interleukin (IL)-4, IL-5 and IL-13 [6]. Dupilumab, a fully human VelocImmune-derived monoclonal antibody [7, 8], blocks the shared receptor component for IL-4 and IL-13, key and central drivers of type 2 inflammation in multiple diseases [9]. In the European Union, dupilumab is indicated as an add-on maintenance treatment in patients aged $\geqslant 12$ years with severe asthma with type 2 inflammation characterised by raised blood eosinophils and/or raised fractional exhaled nitric oxide $\left(F_{\text {eNO }}\right)$ that is inadequately controlled with high-dose inhaled corticosteroids (ICS) plus another medicinal product for maintenance treatment [10-12]. In the phase 3 LIBERTY ASTHMA QUEST study (NCT02414854), add-on dupilumab $200 \mathrm{mg}$ or $300 \mathrm{mg}$ every 2 weeks, versus placebo, significantly reduced severe asthma exacerbations, improved pre-bronchodilator forced expiratory volume in $1 \mathrm{~s}\left(\mathrm{FEV}_{1}\right)$, and was generally well tolerated in the overall population of patients with uncontrolled, moderate-to-severe asthma. Treatment effects were greater in patients with elevated type 2 biomarkers at baseline (blood eosinophils or $F_{\mathrm{eNO}}$ ) [11].

In this non-prespecified post hoc analysis of the phase 3 QUEST study, we assessed efficacy of dupilumab in the population of patients with elevated baseline type 2 biomarker levels (blood eosinophils $\geqslant 150$ cells $\cdot \mu \mathrm{L}^{-1}$ and/or $F_{\text {eNO }} \geqslant 20 \mathrm{ppb}$ ), and stratified them into subgroups by demographic and disease characteristics at baseline (gender, geographical region, body mass index, age, age at asthma onset, medication use, pre-bronchodilator $\mathrm{FEV}_{1}$, number of severe asthma exacerbations in the year before study start, smoking history, blood eosinophil levels, $F_{\text {eNO }}$ levels) to evaluate whether the response to dupilumab was affected by these characteristics. Patients were randomised 2:2:1:1 to receive add-on subcutaneous dupilumab $200 \mathrm{mg}$ or $300 \mathrm{mg}$ or matched-volume placebo every 2 weeks for 52 weeks. Injections were administered during patient study visits until week 12 and could be administered by patients and/or caregivers later. Full details of the study design and methodology have been reported previously [11]. Annualised rate of severe exacerbations during the 52-week treatment period was analysed using negative binomial regression models, with the total number of events occurring during the observation period as the response variable; and treatment group, age, region, baseline eosinophil strata, baseline ICS dose level, number of severe exacerbation events within 1 year prior to the study, subgroup (demographic or disease characteristic if different than the aforementioned) and treatment by subgroup interaction as covariates. Least squares mean change from baseline in pre-bronchodilator $\mathrm{FEV}_{1}$ at week 12 was assessed using mixed-effects models with repeated measures. The model included change from baseline in pre-bronchodilator $\mathrm{FEV}_{1}$ values up to week 12 as response variable; and treatment group, age, patient sex and height, region, baseline eosinophil strata, baseline ICS dose level, visit, treatment-by-visit interaction, baseline pre-bronchodilator $\mathrm{FEV}_{1}$ value, baseline-by-visit interaction, subgroup (demographic or disease characteristic if different than the aforementioned covariates), subgroup-by-treatment interaction, and subgroup-by-treatment-by-visit interaction as covariates.

\section{Shareable abstract (@ERSpublications)}

Dupilumab treatment versus placebo improved exacerbation rate and lung function outcomes in patients with uncontrolled moderate-to-severe asthma and high type 2 biomarkers at baseline, regardless of baseline characteristics in the phase 3 QUEST study https://bit.ly/3yR7MID

Cite this article as: Busse WW, Paggiaro P, Muñoz X, et al. Impact of baseline patient characteristics on dupilumab efficacy in type 2 asthma. Eur Respir J 2021; 58: 2004605 [DOI: 10.1183/13993003.046052020].
\end{abstract}




\section{Subgroup}

Type 2 population

Gender

Male

Female

Region

Asia

Latin America

East Europe

Western countries

BMI $\left(\mathbf{k g} \cdot \mathbf{m}^{-2}\right)$

$<25$

$\geq 25$ to $<30$

$\geq 30$

Age (years)

$<18$

18 to 64

$\geq 65$

Age of onset of asthma (years)

$<18$

18 to 40

$\geq 40$

Medication use

Patients who use ICS and LABA only

Patients who use ICS and LABA and

anti-leukotrienes only

Other

Pre-bronchodilator FEV

$\leq 1.75$

$>1.75$

Number of severe asthma exacerbations

prior to the study

$\leq 1$

$>1$

Smoking history

Never

Former

Blood eosinophils $\left(10^{9}\right.$ per $\left.\mathrm{L}\right)$

$<0.15$

$\geq 0.15$ to $<0.3$

$\geq 0.3$ to $<0.5$

$\geq 0.5$

Baseline $\boldsymbol{F}_{\mathrm{eNO}}(\mathrm{ppb})$

$<25$

$\geq 25$ to $<50$

$\geq 50$

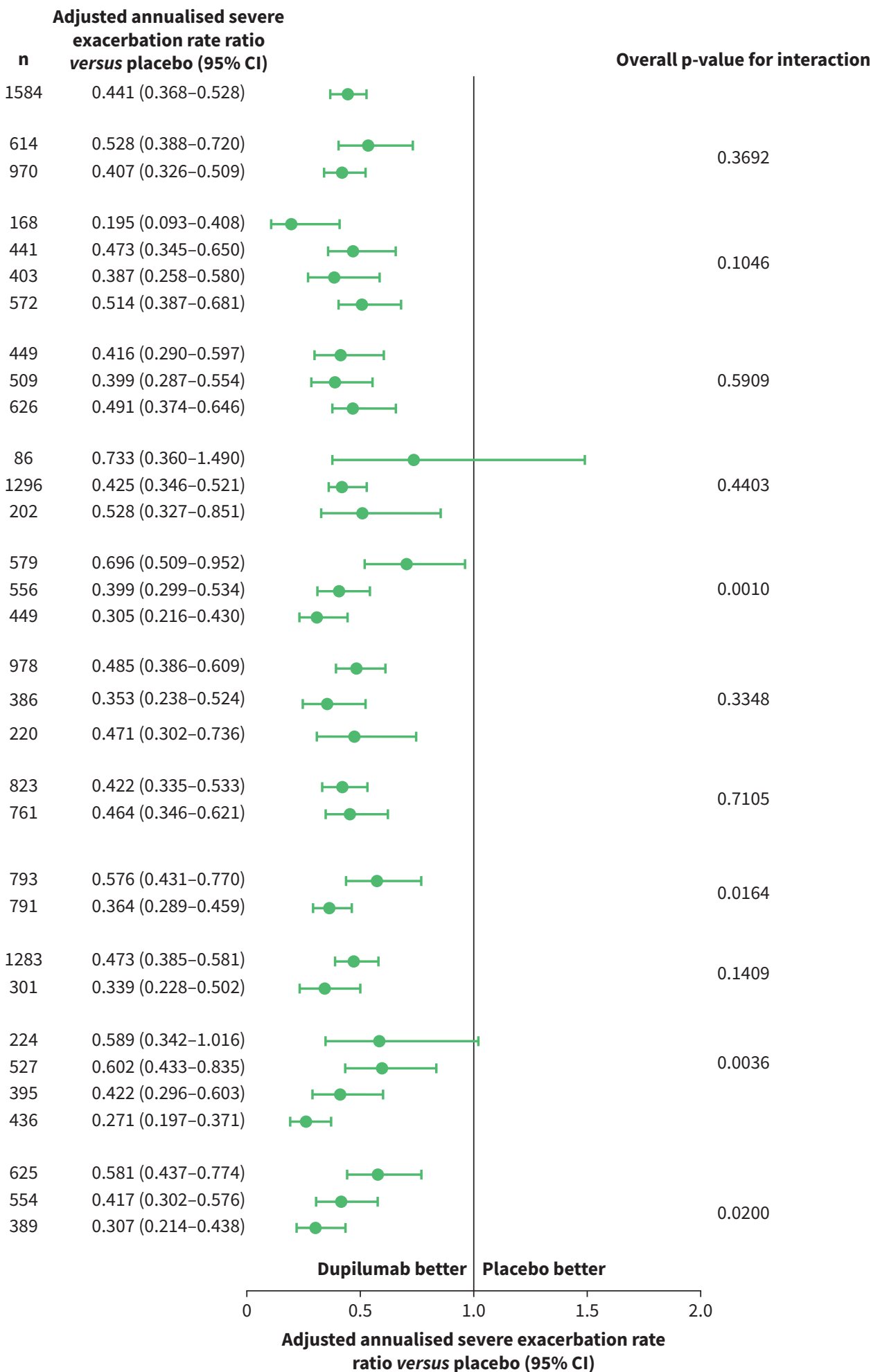

FIGURE 1 a) Adjusted annualised severe exacerbation rate.

Of the 1902 patients randomised in the study, 1584 (dupilumab: 1040; placebo: 544) had baseline blood eosinophils $\geqslant 150$ cells $\cdot \mu \mathrm{L}^{-1}$ or $F_{\mathrm{eNO}} \geqslant 20 \mathrm{ppb}$. Among these patients, demographic and disease characteristics at study initiation were comparable between treatment groups. 


\begin{tabular}{|c|c|c|}
\hline Subgroup & $\mathbf{n}$ & $\begin{array}{c}\text { versus placebo, } \\
\mathrm{L}(95 \% \mathrm{Cl})\end{array}$ \\
\hline Type 2 population & 1533 & $0.15(0.11-0.19)$ \\
\hline \multicolumn{3}{|l|}{ Gender } \\
\hline Male & 593 & $0.18(0.11-0.26)$ \\
\hline Female & 940 & $0.13(0.09-0.18)$ \\
\hline \multicolumn{3}{|l|}{ Region } \\
\hline Asia & 166 & $0.26(0.13-0.39)$ \\
\hline Latin America & 434 & $0.15(0.07-0.23)$ \\
\hline East Europe & 385 & $0.15(0.07-0.24)$ \\
\hline Western countries & 548 & $0.12(0.06-0.19)$ \\
\hline \multicolumn{3}{|l|}{ BMI $\left(\mathrm{kg} \cdot \mathrm{m}^{-2}\right)$} \\
\hline$<25$ & 434 & $0.20(0.11-0.28)$ \\
\hline$\geq 25$ to $<30$ & 496 & $0.13(0.05-0.20)$ \\
\hline$\geq 30$ & 603 & $0.12(0.07-0.18)$ \\
\hline \multicolumn{3}{|l|}{ Age (years) } \\
\hline$<18$ & 83 & $0.33(0.14-0.52)$ \\
\hline 18 to 64 & 1255 & $0.15(0.11-0.20)$ \\
\hline$\geq 65$ & 195 & $0.07(-0.02-0.16)$ \\
\hline \multicolumn{3}{|l|}{ Age of onset of asthma (years) } \\
\hline$<18$ & 562 & $0.11(0.04-0.18)$ \\
\hline 18 to 40 & 535 & $0.17(0.11-0.24)$ \\
\hline$\geq 40$ & 436 & $0.16(0.09-0.23)$ \\
\hline \multicolumn{3}{|l|}{ Medication use } \\
\hline Patients who use ICS and LABA only & 944 & $0.14(0.09-0.19)$ \\
\hline $\begin{array}{l}\text { Patients who use ICS and LABA and } \\
\text { anti-leukotrienes only }\end{array}$ & 376 & $0.17(0.09-0.25)$ \\
\hline Other & 213 & $0.14(0.03-0.24)$ \\
\hline \multicolumn{3}{|l|}{ Pre-bronchodilator $\mathrm{FEV}_{1}$} \\
\hline$\leq 1.75$ & 800 & $0.17(0.11-0.22)$ \\
\hline$>1.75$ & 733 & $0.13(0.07-0.19)$ \\
\hline \multicolumn{3}{|c|}{$\begin{array}{l}\text { Number of severe asthma exacerbations } \\
\text { prior to the study group } 1\end{array}$} \\
\hline$\leq 1$ & 767 & $0.11(0.05-0.17)$ \\
\hline$>1$ & 766 & $0.19(0.13-0.24)$ \\
\hline \multicolumn{3}{|l|}{ Smoking history } \\
\hline Never & 1244 & $0.15(0.11-0.20)$ \\
\hline Former & 289 & $0.13(0.04-0.22)$ \\
\hline \multicolumn{3}{|l|}{ Blood eosinophils ( $10^{9}$ per $\mathrm{L}$ ) } \\
\hline$<0.15$ & 219 & $0.09(-0.02-0.20)$ \\
\hline$\geq 0.15$ to $<0.3$ & 507 & $0.05(-0.02-0.12)$ \\
\hline$\geq 0.3$ to $<0.5$ & 379 & $0.17(0.09-0.25)$ \\
\hline$\geq 0.5$ & 426 & $0.29(0.21-0.37)$ \\
\hline \multicolumn{3}{|l|}{ Baseline $F_{\mathrm{eNO}}(\mathrm{ppb})$} \\
\hline$<25$ & 610 & $0.04(-0.02-0.09)$ \\
\hline$\geq 25$ to $<50$ & 538 & $0.15(0.09-0.22)$ \\
\hline$\geq 50$ & 369 & $0.35(0.26-0.44)$ \\
\hline
\end{tabular}

\section{LS mean difference}

versus placebo,$$
\text { L }(95 \% \mathrm{Cl})
$$

$0.18(0.11-0.26)$

$0.26(0.13-0.39)$

$0.15(0.07-0.23)$

$0.15(0.07-0.24)$

$0.20(0.11-0.28)$

$0.13(0.05-0.20)$

$.12(0.07-0.18)$

$0.33(0.14-0.52)$

$.15(0.11-0.20)$

$0.11(0.04-0.18)$

$0.17(0.11-0.24)$

$0.09-0.19$

$(0.09-0.25)$

$0.17(0.11-0.22)$

$0.13(0.07-0.19)$

$0.11(0.05-0.17)$

$0.13-0.24)$

$0.15(0.11-0.20)$

$0.09(-0.02-0.20)$

$0.05(-0.02-0.12)$

$0.17(0.09-0.25)$

$0.29(0.21-0.37)$

$0.35(0.26-0.44)$
Overall p-value for interaction

0.2200

0.4345

0.2687

0.1241

0.4261

0.8094

0.3689

0.0920

0.6126

0.0002

$<0.0001$

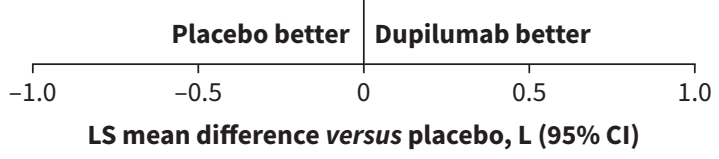

FIGURE 1 b) Least squares (LS) mean difference in the change in pre-bronchodilator forced expiratory volume in $1 \mathrm{~s}\left(\mathrm{FEV}_{1}\right)$ from baseline at week 12 between dupilumab and placebo by baseline patient demographic and disease characteristic subgroup. BMI: body mass index; $F_{\mathrm{eNO}}$ : fractional exhaled nitric oxide; ICS: inhaled corticosteroids; LABA: long-acting $\beta 2$-agonists; ppb: parts per billion.

Dupilumab versus placebo reduced the annualised rate of severe exacerbations, irrespective of demographic or disease characteristics at baseline (figure 1a). In general, efficacy of dupilumab was comparable between demographic and disease characteristic subgroups. Dupilumab efficacy was greater in patients with $>1$ 
exacerbation in the year prior to study initiation $(\mathrm{p}=0.0164)$, and age at asthma onset $>18$ years $(p=0.0010)$. Dupilumab had a greater treatment effect in patients with higher baseline blood eosinophil concentrations ( $\mathrm{p}=0.0036)$ and $F_{\text {eNO }}$ levels $(\mathrm{p}=0.0200)$ in line with previous observations [11].

In this post hoc analysis, dupilumab improved pre-bronchodilator $\mathrm{FEV}_{1} 12$ weeks after treatment initiation in all demographic and disease characteristic subgroups examined (figure 1b). No significant treatment-by-subgroup interactions were detected, with the exception of baseline blood eosinophil concentration $(\mathrm{p}=0.0002)$ and $F_{\text {eNO }}$ levels $(\mathrm{p}<0.0001)$, suggesting a comparable treatment benefit for all patients, irrespective of their demographic or disease characteristics.

Our findings suggest that in this population of patients with elevated type 2 biomarkers at baseline, dupilumab reduced the annualised rate of severe exacerbations and improved pre-bronchodilator $\mathrm{FEV}_{1}$ consistently across most patient demographic and disease characteristics at baseline. This included patients with differing gender, geographical region, body mass index, age, age at asthma onset, medication use, pre-bronchodilator $\mathrm{FEV}_{1}$, number of severe asthma exacerbations in the year before study start, smoking history, blood eosinophil levels, and $F_{\text {eNO }}$ levels. Previous studies have found the efficacy of some asthma treatments to vary depending on patient demographics, for example age and age of asthma onset, with limited efficacy observed in patients with asthma onset $<18$ years [4, 5]. Although patients with differing age at asthma onset and exacerbation history showed variations in the degree to which the exacerbation rate was reduced, dupilumab was efficacious in reducing severe exacerbations versus placebo in all demographic and disease characteristic subgroups evaluated, including those in whom asthma started before age 18 years. Benefits of dupilumab on patient lung function were not impacted by demographic and disease characteristics. Taken together, these findings add to the body of knowledge guiding treatment decisions for asthma patients. Current EAACI guidelines recommend the use of dupilumab as an add-on treatment for adult and adolescent patients with severe uncontrolled asthma with a type 2 phenotype [13-15]. This analysis supports these recommendations, demonstrating that dupilumab treatment is efficacious in all patients with moderate-to-severe, type 2 asthma, regardless of demographics or disease characteristics.

In conclusion, the response to dupilumab treatment in patients with uncontrolled, moderate-to-severe, type 2 asthma was unaffected by patient demographic or disease characteristics at baseline.

William W. Busse ${ }^{1}$, Pierluigi Paggiaro ${ }^{2}$, Xavier Muñoz ${ }^{3}$, Thomas B. Casale ${ }^{4}$, Mario Castro ${ }^{5}$, G. Walter Canonica $\oplus^{6}$, Jo A. Douglass ${ }^{7,8}$, Yuji Tohda ${ }^{9}$, Nadia Daizadeh ${ }^{10}$, Benjamin Ortiz ${ }^{11}$ and Nami Pandit-Abid ${ }^{12}$

${ }^{1}$ UW Allergy, Pulmonary and Critical Care Medicine, University of Wisconsin School of Medicine and Public Health, Madison, WI, USA. ${ }^{2}$ University of Pisa, Pisa, Italy. ${ }^{3}$ Pneumology Dept, Hospital Vall d'Hebron, Barcelona, Spain. ${ }^{4}$ University of South Florida, Tampa, FL, USA. ${ }^{5}$ University of Kansas School of Medicine, Kansas City, KS, USA. ${ }^{6}$ Humanitas University and Research Hospital-IRCCS, Milan, Italy. ${ }^{7}$ Royal Melbourne Hospital, Melbourne, Australia. ${ }^{8}$ The University of Melbourne, Melbourne, Australia. ${ }^{9}$ Faculty of Medicine, Kindai University Hospital, Osakasayama, Japan. ${ }^{10}$ Sanofi, Cambridge, MA, USA. ${ }^{11}$ Regeneron Pharmaceuticals, Inc., Tarrytown, NY, USA. ${ }^{12}$ Sanofi, Bridgewater, NJ, USA.

Corresponding author: William W. Busse (wwb@medicine.wisc.edu)

Acknowledgements: Medical writing/editorial assistance provided by Grace Manley, PhD, of Excerpta Medica, funded by Sanofi Genzyme and Regeneron Pharmaceuticals, Inc.

This study is registered with ClinicalTrials.gov with identifier number NCT0414854.

Conflict of interest: W.W. Busse is a consultant for AstraZeneca, Genentech, GlaxoSmithKline, Novartis, Regeneron Pharmaceuticals, Inc. and Sanofi; and is on the data safety monitoring board for Boston Scientific. P. Paggiaro received research grants and is an advisory board member for AstraZeneca, Chiesi, GlaxoSmithKline, Novartis and Sanofi. X. Muñoz is a speaker, scientific advisor for and has received clinical trial investigator fees from AstraZeneca, Boehringer Ingelheim, Chiesi, Faes Farma, GlaxoSmithKline, Menarini, Mundipharma, Novartis and Teva. T.B. Casale received research support from American Lung Association, Genentech, NIH, Novartis, PCORI and Sanofi; is a consultant for AstraZeneca, Boehringer Ingelheim, Genentech, Novartis and Regeneron Pharmaceuticals, Inc.; is on the speakers bureau of Genentech. M. Castro received research support from American Lung Association, AstraZeneca, GlaxoSmithKline, NIH, Novartis, PCORI, Pulmatrix, sanofi-aventis and Shionogi; is a 
consultant for Genentech, Novartis, sanofi-aventis and Teva; received speaker fees from AstraZeneca, Genentech, GlaxoSmithKline, Regeneron Pharmaceuticals, Inc., Sanofi and Teva; received royalties from Elsevier. G.W. Canonica received speaker fees and is an advisory board member of ALK, AstraZeneca, Boehringer Ingelheim, GlaxoSmithKline, HAL Allergy, Menarini, Mundipharma, Novartis, Regeneron Pharmaceuticals, Inc., Sanofi, Stallergenes Greer and Uriach. J.A. Douglass received research funding, speaker fees and is an advisory board member of AstraZeneca, GlaxoSmithKline, Novartis and Sanofi. Y. Tohda is a consultant for AstraZeneca, Kyorin Pharmaceuticals and Sanofi. N. Daizadeh is an employee of and may hold stock and/or stock options in Sanofi. B. Ortiz is an employee and shareholder of Regeneron Pharmaceuticals, Inc. N. Pandit-Abid is an employee of and may hold stock and/or stock options in Sanofi.

Support statement: Research sponsored by Sanofi and Regeneron Pharmaceuticals, Inc. Funding information for this article has been deposited with the Crossref Funder Registry.

\section{References}

1 Chung KF, Wenzel SE, Brozek JL, et al. International ERS/ATS guidelines on definition, evaluation and treatment of severe asthma. Eur Respir J 2014; 43: 343-373.

2 Zein JG, Denson JL, Wechsler ME. Asthma over the adult life course: gender and hormonal influences. Clin Chest Med 2019; 40: 149-161.

3 Khalid F, Holguin F. A review of obesity and asthma across the life span. J Asthma 2018; 55: 1286-1300.

4 Harrison TW, Chanez P, Menzella F, et al. Onset of effect and impact on health-related quality of life, exacerbation rate, lung function, and nasal polyposis symptoms for patients with severe eosinophilic asthma treated with benralizumab (ANDHI): a randomised, controlled, phase 3b trial. Lancet Respir Med 2021; 9: 260-274.

5 Sposato B, Scalese M, Latorre M, et al. Effects of omalizumab in severe asthmatics across ages: a real life Italian experience. Respir Med 2016; 119: 141-149.

6 Fahy JV. Type 2 inflammation in asthma-present in most, absent in many. Nat Rev Immunol 2015; 15: 57-65.

7 Macdonald LE, Karow M, Stevens S, et al. Precise and in situ genetic humanization of $6 \mathrm{Mb}$ of mouse immunoglobulin genes. Proc Natl Acad Sci USA 2014; 111: 5147-5152.

8 Murphy AJ, Macdonald LE, Stevens S, et al. Mice with megabase humanization of their immunoglobulin genes generate antibodies as efficiently as normal mice. Proc Natl Acad Sci USA 2014; 111: 5153-5158.

9 Gandhi NA, Pirozzi G, Graham NMH. Commonality of the IL-4/IL-13 pathway in atopic diseases. Expert Rev Clin Immunol 2017; 13: 425-437.

10 Wenzel S, Castro M, Corren J, et al. Dupilumab efficacy and safety in adults with uncontrolled persistent asthma despite use of medium-to-high-dose inhaled corticosteroids plus a long-acting $\beta 2$ agonist: a randomised double-blind placebo-controlled pivotal phase 2b dose-ranging trial. Lancet 2016; 388: 31-44.

11 Castro M, Corren J, Pavord ID, et al. Dupilumab efficacy and safety in moderate-to-severe uncontrolled asthma. N Engl J Med 2018; 378: 2486-2496.

12 Rabe KF, Nair P, Brusselle G, et al. Efficacy and safety of dupilumab in glucocorticoid-dependent severe asthma. N Engl J Med 2018; 378: 2475-2485.

13 Agache I, Akdis CA, Akdis M, et al. EAACI Biologicals Guidelines-Recommendations for severe asthma. Allergy 2021; 76: 14-44.

14 Agache I, Song Y, Rocha C, et al. Efficacy and safety of treatment with dupilumab for severe asthma: a systematic review of the EAACl guidelines-Recommendations on the use of biologicals in severe asthma. Allergy 2020; 75: 1058-1068.

15 Doroudchi A, Pathria M, Modena BD. Asthma biologics: comparing trial designs, patient cohorts and study results. Ann Allergy Asthma Immunol 2020; 124: 44-56. 